\title{
Pulpa Dentaria Sana vs. Pulpitis Reversible: Caracterización Estereológica de Fibroblastos
}

\author{
Healthy Normal Dental Pulp vs. Reversible Pulpitis: \\ Stereological Characterization of Fibroblasts
}

\author{
Erick Trujillo*; Rolando Morales ${ }^{* *}$ \& Ignacio Roa ${ }^{* * * * * * * * * * * * * *}$
}

TRUJILLO, E.; MORALES, R. \& ROA, I. Pulpa dentaria sana vs. pulpitis reversible: caracterización estereológica de fibroblastos. Int. J. Morphol., 34(3):945-949, 2016.

RESUMEN: La reacción y reparación de la dentina depende del número de células presentes en la pulpa, dentro de éstas fibroblastos. Los métodos diseñados para obtener una estimación fiable de la cantidad de elementos celulares de la pulpa han sido subjetivos y sesgados, sobre todo al evaluar los cambios cuantitativos y potencial capacidad reparadora en presencia de caries. El objetivo fue estimar y comparar cuantitativamente las densidades de número, volumen y superficie de fibroblastos en pulpas sanas y con diagnóstico de pulpitis reversible producto de caries en dientes humanos jóvenes. Se utilizaron dientes premolares humanos obtenidos de exodoncias, divididos en un grupo sano y cariado, los cuales fueron fijados y posteriormente descalcificados con ácido nítrico al $5 \%$. Siguiendo el protocolo del orientator se obtuvieron 5 secciones de $5 \mu \mathrm{m}$ teñidas por H-E de cada diente. Se aplicó el recuento estereológico de los fibroblastos pulpares (FP) con el test multipropósito M42. Se estimaron las densidades de número (Nv), volumen (Vv) y superficie $(\mathrm{Sv})$, y calcularon las Medias $( \pm \mathrm{DE})$ por diente, y Medias $( \pm \mathrm{EE})$ por grupo. Las diferencias entre grupos se analizaron mediante la prueba $\mathrm{T}$, con un valor $\mathrm{p} \leq 0,05$ de significación estadística. En dientes sanos, la Media ( \pm EE) para Nv de FP fue $0,393 \times 10^{5} / \mathrm{mm}^{3}\left( \pm 0,020 \times 10^{5} /\right.$ $\left.\mathrm{mm}^{3}\right)$, para Vv $15,467 \%( \pm 1,334 \%)$ y para Sv $16,330 \mathrm{~mm}^{2} / \mathrm{mm}^{3}\left( \pm 1,274 \mathrm{~mm}^{2} / \mathrm{mm}^{3}\right)$. En dientes cariados, la $\mathrm{Nv}$ fue $0,447 \mathrm{x} 10^{5} / \mathrm{mm}^{3}$ $\left( \pm 0,019 \times 10^{5} / \mathrm{mm}^{3}\right)$, la Vv 20,171\% $( \pm 1,213 \%)$ y la Sv $20,150 \mathrm{~mm}^{2} / \mathrm{mm}^{3}\left( \pm 1,447 \mathrm{~mm}^{2} / \mathrm{mm}^{3}\right)$. Al comparar las Nv, los FP del grupo con caries aumentaron significativamente $(\mathrm{p}=0,047)$, al igual que la $\mathrm{Vv}(\mathrm{p}=0,0105)$ y $\mathrm{Sv}(\mathrm{p}=0,013)$. Existe un aumento del número de FP en los dientes con pulpitis reversible, lo que condicionaría su capacidad de respuesta. La metodología empleada puede ser aplicable para determinar el comportamiento pulpar y cuantificar variables de respuesta odontoblástica en tratamientos restauradores atraumáticos de manera imparcial y reproducible.

PALABRAS CLAVE: Estereología; Pulpa dentaria; Fibroblastos.

\section{INTRODUCCIÓN}

Es bien sabido que las bacterias son la principal causa de infección e inflamación pulpar (Hahn \& Liewehr, 2007). Si bien esta se encuentra protegida por una serie de tejidos duros, estos pueden ser vulnerados por las bacterias o sus productos (Fuks et al., 2016), resultando en la infección de la pulpa. Este proceso dinámico depende tanto de los patógenos invasores como de las respuestas del huésped, que incluyen inflamación y respuesta inmune (Núñez \& García Bacallao, 2010).

Los fibroblastos pulpares (FP), son la población celular más numerosa dentro de la pulpa, teniendo por fun- ción la síntesis y degradación de colágeno, así como de los demás elementos de la matriz extracelular (Trowbridge et al., 2002; Li \& Wang, 2011). Asimismo ha sido reportada la síntesis de mediadores de inflamación y citoquinas en respuesta a componentes bacterianos (Nakanishi et al., 2011), contribuyendo además a la inmunidad innata (Takeuchi \& Akira, 2010). Si bien su función principal es secretora, ante injuria pulpar, estas células son las primeras en ir en reemplazo de los odontoblastos destruidos y diferenciarse a estos para suplir su función (Vavpotic et al., 2009; Nakanishi et al.), por lo cual los FP pueden ser considerados células odontoprogenitoras (Liwei et al., 2016).

\footnotetext{
* Escuela de Medicina, Universidad de Talca, Talca, Chile.

** Universidad de Viña del Mar, Viña del Mar, Chile.

*** Unidad de Morfología, Departamento de Ciencias Básicas Biomédicas, Facultad de Ciencias de la Salud, Universidad de Talca, Talca, Chile.

***** Programa de Doctorado en Ciencias Morfológicas, Facultad de Medicina, Universidad de La Frontera, Temuco, Chile.

******* Becario CONICYT-PCHA/Doctorado Nacional/2015-21150235.
} 
Por otra parte estudios in vitro indican el importante rol que presentan los FP en la activación del sistema del complemento, poniendo de manifiesto claramente la función en la destrucción de las bacterias cariogénicas, tales como S. mutans y S. sanguinis (Jeanneau et al., 2015). Así como, estos contribuyen significativamente a la regeneración de los tejidos mediante el reclutamiento de células progenitoras de pulpa a través de la activación del complemento (Chmilewsky et al., 2014).

Cambios cuantificables en la pulpa dental han sido descritos anteriormente, estimándose la cantidad y densidad de odontoblastos en un estado de salud o reacción pulpar (Morales et al., 2014), pero no así de los FP. Hasta hace poco, los métodos para obtener estimaciones fiables sobre la cantidad de FP en la pulpa dental han sido insuficientes. La aplicación de la estereología es un método fiable para estimar la cantidad de células en un tejido biológico determinado (Mandarim-de-Lacerda et al., 2010; Salgado et al., 2011; Trujillo et al., 2011; Sentut et al., 2012).

El objetivo del presente estudio, fue estimar y comparar cuantitativamente la densidad de número y volumen y superficie de fibroblastos en pulpas sanas y con diagnóstico clínico de pulpitis reversible producto de caries en dientes humanos jóvenes.

\section{MATERIAL Y MÉTODO}

Fue realizado un estudio de corte transversal, con muestreo aleatorio simple, correspondiente a dientes premolares humanos, clínicamente sanos o con diagnóstico de pulpitis reversible producida por caries, obtenidos de exodoncias practicadas en servicios públicos de la Séptima Región y la Clínica de Urgencias e Internado de Cirugía de la Escuela de Odontología, Universidad de Talca, entre Marzo y Agosto del año 2015. Fueron incluidos dientes con indicación de exodoncia por necesidad de tratamiento ortodóntico, de pacientes con edades entre 15 y 20 años. Se excluyeron dientes con atrición evidente, restauraciones de cualquier tipo o extensión, o cuya exodoncia implicara osteotomía u odontosección. El protocolo de investigación fue revisado y aprobado por el Comité de Ética Científica de la Universidad de Talca (\#00184).

La muestra estuvo constituida por 10 dientes, divididos en 2 grupos: control (dientes sanos sin caries) $(n=5)$ y cariados (dientes con diagnóstico de pulpitis reversible) $(\mathrm{n}=5)$. Inmediatamente posterior a su exodoncia, estos fueron fijados con formalina tamponada al $10 \%$ por $24 \mathrm{~h}, \mathrm{y}$ luego se sometieron a inmersión en ácido nítrico al $5 \%$ para su descalcificación durante 14 días. Los dientes se orientaron en los bloques de parafina de modo que se pudieran cortar y obtener secciones mesio-distales paralelas a su eje longitudinal (Morales et al.).

Se realizaron cortes longitudinales de cada diente, con el fin de exponer la pulpa hacia una de las caras libre del taco para facilitar su corte, siguiendo el protocolo del orientator (Mattfeldt et al., 1990). Se obtuvieron 5 cortes de cada diente, con un grosor de $5 \mu \mathrm{m}$ para ser teñidos mediante H-E. Donde fue confirmado histológicamente el diagnóstico de pulpitis reversible. En las muestras de ambos grupos, se identificaron y analizaron 5 campos por cada corte, totalizando 125 campos por diente. Las muestras fueron observadas en un microscopio óptico (Carl Zeiss ${ }^{\circledast}$ Primo Star) con una cámara $\left(\mathrm{Canon}^{\circledR}\right.$ modelo EOS Rebel Xsi) integrada. Las imágenes obtenidas se proyectaron en un monitor Samsung ${ }^{\oplus}$ HD, y se aplicó el recuento estereológico con el test multipropósito M42 (Mandarim-de-Lacerda, 2003).

El análisis estereológico consistió en la estimación de la densidad de número $(\mathrm{Nv})$, densidad de volumen $(\mathrm{Vv})$ y densidad de superficie (Sv). Se calculó la Media y desviación estándar para cada diente, y la Media y error estándar para cada grupo. En todos los casos se utilizó un intervalo de confianza de $95 \%$. Las diferencias entre los grupos se analizaron mediante la prueba $\mathrm{T}$ para variables paramétricas independientes. Los valores de Medias se consideraron estadísticamente significativos con un valor $\mathrm{p} \leq 0,05$. El análisis estadístico se realizó con el programa STATA versión 11.0 y los datos fueron graficados con el programa GraphPad Prism 5.

\section{RESULTADOS}

En el grupo control, la Nv Media de fibroblastos en el tejido pulpar fue $0,393 \times 10^{5} / \mathrm{mm}^{3}$, con un error estándar de $\pm 0,020 \times 10^{5} / \mathrm{mm}^{3}$. La Vv Media de fibroblastos fue 15,467 $\%$ con un error estándar de $\pm 1,334 \%$. La Media del Sv de fibroblastos fue $16,330 \mathrm{~mm}^{2} / \mathrm{mm}^{3}$ con un error estándar de $\pm 1,274 \mathrm{~mm}^{2} / \mathrm{mm}^{3}$. El detalle de la Media ( \pm DE) para las $\mathrm{Nv}, \mathrm{Vv}$ y Sv de los fibroblastos de cada diente sano se observa en la Figura 1 (A-C).

En el grupo caries, la Nv Media de fibroblastos del tejido pulpar fue $0,447 \times 10^{5} / \mathrm{mm}^{3}$, con un error estándar de $\pm 0,019 \times 10^{5} / \mathrm{mm}^{3}$. La Vv Media fue $20,171 \%$ con un error estándar de $\pm 1,213 \%$. La Media del Sv fue $20,150 \mathrm{~mm}^{2} /$ $\mathrm{mm}^{3}$ con un error estándar de $\pm 1,447 \mathrm{~mm}^{2} / \mathrm{mm}^{3}$. El detalle de la Media ( \pm DE) para las Nv, Vv y Sv de los fibroblastos de cada diente con caries se observa en la Figura 1 (A-C). 


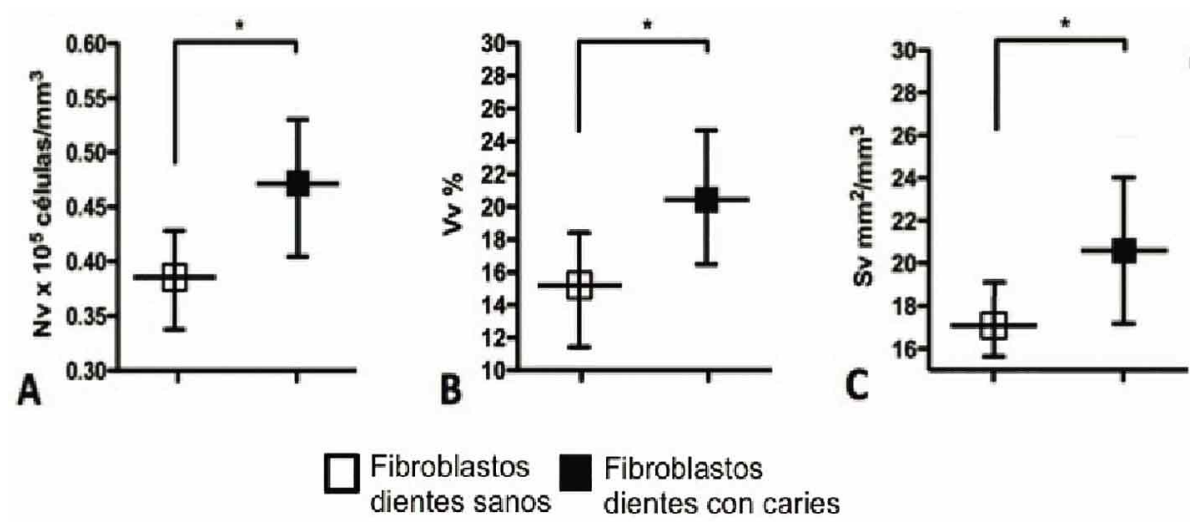

Fig. 1. Detalle de Media y DE para las variables Nv (A), Vv (B) y Sv (C) de fibroblastos del grupo sano y con caries.

$\mathrm{Al}$ comparar la $\mathrm{Nv}$ de fibroblastos, en el grupo de dientes con caries el aumento observado fue significativo $(\mathrm{p}=0,047)$ con respecto al grupo sano, al igual que lo observado al comparar la $\mathrm{Vv}(\mathrm{p}=0,0105)$ y $\mathrm{Sv}(\mathrm{p}=0,013)$.

\section{DISCUSIÓN}

La vitalidad y capacidad reparativa en el diente se debe a la presencia de la pulpa, gracias a sus componentes vasculares y nerviosos, los cuales en conjunto a los distintos elementos celulares mantiene su fisiología normal. Además de encargarse de la producción de dentina durante etapas de maduración, así como, reacción a injurias o estímulos, ya sean traumáticos o infecciosos como la caries (Bruno et al., 2010).

Al análisis de los parámetros estereológicos para FP, es posible observar también, un aumento estadísticamente significativo en $\mathrm{Nv}$, situación que se repite para el parámetro de $\mathrm{Vv}$ donde hay un aumento en el porcentaje promedio de fibroblastos cuando existían caries asociada al diente. Esto mismo se da en Sv, donde es posible afirmar un aumento en la superficie total de fibroblastos en dientes con caries, comparados con la superficie promedio ocupada por fibroblastos en dientes sanos, aumento que es estadísticamente significativo.

Estos resultados podrian ser explicados según lo expresado por Eslaminejad et al. (2010), quienes relatan que los FP son capaces de proliferar rápidamente y formar una monocapa cuando son expuestos a condiciones adversas como injurias y productos bacterianos, así como también, en condiciones experimentales. Por otra parte, en procesos de reparación pulpar iniciales y frente a ataque de caries, habría un aumento cuantitativo de FP, indu- cidos por MAP kinasas (Gruber et al., 2004); además, se ha visto que durante procesos de inflamación pulpar, los $\mathrm{FP}$, son una de las poblaciones celulares que más se incrementan, junto con la masiva migración de células inflamatorias y la proliferación de células indiferenciadas (Goldberg et al., 2008), debido en parte a la secreción de factores de crecimiento implicados en la activación y diferenciación de células progenitoras, por parte de FP afectados (About, 2011).

Durante los procesos de inflamación pulpar hay un incremento general en el número de células pulpares, tanto de odontoblastos como de fibroblastos (Goldberg et al.; Bjorndal, 2002), definido por mediadores de la inflamación como prostaglandinas y leucotrienos, que favorecen una reorientación del fenotipo celular y promueven la transdiferenciación de algunas células inflamatorias en odontoblastos, lo cual también, ocurre en el caso de los fibroblastos, que presentan una estructura determinada que también les permite un potencial de transdiferenciación posterior a su multiplicación (Goldberg et al.).

Frente al ataque de caries, la pulpa dental presentará cambios celulares y estructurales, que se relacionarán directamente con su extensión y avance (Vitalariu et al., 2005), que se podría traducir en una adecuada respuesta, diferenciación y proliferación de otros fenotipos celulares de la pulpa con potencial hacia células de tipo odontoblasto, lo cual sería posible en una condición de pulpitis reversible como la analizada, con una lesión cariosa dentinaria de baja latencia. La capacidad de reparación frente a una injuria, no sólo dependerá del número de elementos celulares (About), sino que de otros factores tales como inflamación pulpar, respuesta inmediata o acumulativa, además de factores externos tales como espesor de la dentina, presencia de bacterias, de un material de restauración y su toxicidad (Sentut et al.). 
La subjetividad y sesgo al analizar cambios en las estructuras biológicas es un problema al utilizar métodos de tipo cualitativo (Warfvinge, 1987). Con el fin de eliminar estos elementos que distorsionan los resultados y así poder cuantificar las estructuras morfológicas de un modo uniforme, reproducible e imparcial, es necesario la utilización de un muestreo aleatorio y el uso de herramientas estereológicas (Morales et al.). El uso de los principios estereológicos ha sido utilizado previamente en estudios en pulpa dental con el fin de analizar la pérdida de odontoblastos con la edad (Murray et al., 2002) o ver los cambios en la pulpa dental afectada por periodontitis (Heidari et al., 2013), así como cambios en dientes de ratas sometidas a movimientos ortodónticos (Santamaria et al., 2006). El análisis de los resultados obtenidos demuestra la capacidad de respuesta reparativa pulpar, por medio del aumento de FP, gracias a la utilización de análisis estereológico.

TRUJILLO, E.; MORALES, R. \& ROA, I. Healthy normal dental pulp vs. reversible pulpitis: stereological characterization of fibroblasts. Int. J. Morphol., 34(3):945-949, 2016.

SUMMARY: Dentine reaction and repair depends on the number of cells present in the pulp, within these fibroblasts. The methods designed to obtain a reliable estimate of the amount of cellular elements of the dental pulp have been subjective and biased, especially when evaluating quantitative changes and potential reparative capacity in the presence of caries. The aim of this study was to estimate and quantitatively compare with stereological tools, number, density, volume and surface of fibroblasts in healthy teeth and reversible pulpitis diagnosis due to caries. We obtained premolar teeth from human tooth extractions, divided into healthy and carious groups, which were fixed and decalcified with $5 \%$ nitric. Following the orientator protocol we obtained 5 sections of $5 \mu \mathrm{m}$ from each tooth which were stained by H-E. The stereological counting of pulp fibroblasts (FP) with M42 multipurpose test was applied. Number densities (Nv), volume ( $\mathrm{Vv}$ ) and surface (Sv) were estimated, and calculated the means $( \pm S D)$ for a tooth, and Mean $( \pm S E)$ per group. Differences between groups were analyzed by t-test, $p$ $\leq 0.05$ a statistically significant value. In healthy teeth, the mean $\left( \pm\right.$ SE) for $\mathrm{Nv}$ FP was $0.393 \times 10^{5} / \mathrm{mm}^{3}\left( \pm 0.020 \times 10^{5} / \mathrm{mm}^{3}\right)$, Vv $15.467 \%( \pm 1.334 \%)$ and $\mathrm{Sv} 16.330 \mathrm{~mm}^{2} / \mathrm{mm}^{3}\left( \pm 1.274 \mathrm{~mm}^{2} /\right.$ $\left.\mathrm{mm}^{3}\right)$. In decayed teeth, it was $0.447 \times 10^{5} \mathrm{Nv} / \mathrm{mm}^{3}( \pm 0.019 \times 105 /$ $\left.\mathrm{mm}^{3}\right)$, the $\mathrm{Vv} 20.171 \%( \pm 1.213 \%)$ and $\mathrm{Sv} 20.150 \mathrm{~mm}^{2} / \mathrm{mm}^{3}( \pm$ $\left.1.447 \mathrm{~mm}^{2} / \mathrm{mm}^{3}\right)$. Comparing Nv, the FP carious group increased significantly $(p=0.047)$, as $\mathrm{Vv}(\mathrm{p}=0.0105)$ and $\mathrm{Sv}(\mathrm{p}=0.013)$. There is an increased number of FP teeth with reversible pulpitis, which would determine their responsiveness. The methodology can be applied to determine the pulp behavior and odontoblast quantify response variables in impartially and reproducible atraumatic restorative treatments.

KEY WORDS: Stereology; Dental pulp; Fibroblasts.

\section{REFERENCIAS BIBLIOGRÁFICAS}

About, I. Dentin regeneration in vitro: the pivotal role of supportive cells. Adv. Dent. Res., 23(3):320-4, 2011.

Bjorndal, L. Dentin and pulp reactions to caries and operative treatment: biological variables affecting treatment outcome. Endod. Topics, 2(1):10-23, 2002.

Bruno, K. F.; Silva, J. A.; Silva, T. A.; Batista, A. C.; Alencar, A. H. \& Estrela, C. Characterization of inflammatory cell infiltrate in human dental pulpitis. Int. Endod. J., 43(11):1013-21, 2010.

Chmilewsky, F.; Jeanneau, C.; Laurent, P. \& About, I. Pulp fibroblasts synthesize functional complement proteins involved in initiating dentin-pulp regeneration. Am. J. Pathol., 184(7):1991-2000, 2014.

Eslaminejad, M. B.; Vahabi, S.; Shariati, M. \& Nazarian, H. In vitro growth and characterization of stem cells from human dental pulp of deciduous versus permanent teeth. J. Dent. (Tehran), 7(4):185-95, 2010.

Fuks, A. B.; Hebling, J. \& Costa de Souza, C. A. The Primary Pulp: Developmental and Biomedical Background. In: Fuks, A. B. \& Peretz, B. (Eds.). Pediatric Endodontics. Current Concepts in Pulp Therapy for Primary and Young Permanent Teeth. Heidelberg, Springer International Publishing, 2016.

Goldberg, M.; Farges, J. C.; Lacerda-Pinheiro, S.; Six, N.; Jegat, N.; Decup, F.; Septier, D.; Carrouel, F.; Durand, S.; ChaussainMiller, C.; Denbesten, P.; Veis, A. \& Poliard, A. Inflammatory and immunological aspects of dental pulp repair. Pharmacol. Res., 58(2):137-47, 2008.

Gruber, R.; Jindra, C.; Kandler, B.; Watzak, G.; Fischer, M. B. \& Watzek, G. Proliferation of dental pulp fibroblasts in response to thrombin involves mitogen-activated protein kinase signalling. Int. Endod. J., 37(2):145-50, 2004.

Hahn, C. L. \& Liewehr, F. R. Relationships between caries bacteria, host responses, and clinical signs and symptoms of pulpitis. J. Endod., 33(3):213-9, 2007.

Heidari, Z.; Saberi, E. A.; Mahmoudzadeh-Sagheb, H.; FarhadMollashahi, N. \& Zadfatah, F. Stereological analysis of the dental pulp in patients with advanced periodontitis. Zahedan J. Res. Med. Sci., 15(7):44-9, 2013.

Jeanneau, C.; Rufas, P.; Rombouts, C.; Giraud, T.; Dejou, J. \& About, I. Can pulp fibroblasts kill cariogenic bacteria? Role of complement activation. J. Dent. Res., 94(12):1765-72, 2015.

Li, B. \& Wang, J. H. Fibroblasts and myofibroblasts in wound healing: force generation and measurement. J. Tissue Viability, 20(4):108-20, 2011. 
Liwei, Z.; Chenglin, W. \& Ling, Y. Tooth Development: Embryology of the Craniofacial Tissues. In: Xuedong, Z. (Ed.). Dental Caries. Principles and Management. Heidelberg, SpringerVerlag, 2016.

Mandarim-de-Lacerda, C. A. Stereological tools in biomedical research. An. Acad. Bras. Cienc., 75(4):469-86, 2003.

Mandarim-de-Lacerda, C. A.; Fernandes-Santos, C. \& Aguila, M. B. Image analysis and quantitative morphology. Methods Mol. Biol., 611:211-25, 2010.

Mattfeldt, T.; Mall, G.; Gharehbaghi, H. \& Möller, P. Estimation of surface area and length with the orientator. J. Microsc., 159(Pt. 3):301-17, 1990.

Morales, R.; Trujillo, E. \& Cantín, M. Stereological characterization of odontoblasts in normal healthy and reversible pulpitis in human dental pulps. Int. J. Morphol., 32(1):154-60, 2014.

Murray, P. E.; Matthews, J. B.; Sloan, A. J. \& Smith, A. J. Analysis of incisor pulp cell populations in Wistar rats of different ages. Arch. Oral Biol., 47(10):709-15, 2002.

Nakanishi, T.; Takegawa, D.; Hirao, K.; Takahashi, K.; Yumoto, H. \& Matsuo, T. Roles of dental pulp fibroblasts in the recognition of bacterium-related factors and subsequent development of pulpitis. Jpn. Dent. Sci. Rev., 47(2):161-6, 2011.

Núñez, D. P. \& García Bacallao, L. Bioquímica de la caries dental. Rev. Haban. Cienc. Méd., 9(2):156-66, 2010.

Salgado, A. G.; Vásquez, B. \& del Sol, M. Stereology of the human thyroid gland. Int. J. Morphol., 29(2):562-7, 2011.

Santamaria, M. Jr.; Milagres, D.; Stuani, A. S.; Stuani, M. B. \& Ruellas, A. C. Initial changes in pulpal microvasculature during orthodontic tooth movement: a stereological study. Eur. J. Orthod., 28(3):217-20, 2006.

Sentut, T.; Kirzioglu, Z.; Gökcimen, A.; Aslan, H. \& Erdogan, Y. Quantitative analysis of odontoblast cells in fluorotic and nonfluorotic primary tooth pulp. Turk. J. Med. Sci., 42(2):3517, 2012.

Takeuchi, O. \& Akira, S. Pattern recognition receptors and inflammation. Cell, 140(6):805-20, 2010.

Trowbridge, H.; Kim, S. \& Suda, H. Estructura y Funciones del Complejo Dentino-Pulpar. En: Cohen, S. \& Burns, R. (Eds.). Vías de la Pulpa. 8a ed. Madrid, Mosby, 2002.

Trujillo, E.; Vásquez, B. \& del Sol, M. Stereologic characteristics of the liver of rats (Rattus norvergicus) submitted to ovariectomy-induced menopause. Int. J. Morphol., 29(4):1470$8,2011$.
Vavpotic, M.; Turk, T.; Martincic, D. S. \& Balazic, J. Characteristics of the number of odontoblasts in human dental pulp postmortem. Forensic Sci. Int., 193(1-3):122-6, 2009.

Vitalariu, A.; Caruntu, I. D. \& Bolintineanu, S. Morphological changes in dental pulp after the teeth preparation procedure. Rom. J. Morphol. Embryol., 46(2):131-6, 2005.

Warfvinge, J. Morphometric analysis of teeth with inflamed pulp. J. Dent. Res., 66(1):78-83, 1987.

\author{
Dirección para Correspondencia: \\ Ignacio Roa Henríquez \\ Unidad de Morfología \\ Departamento de Ciencias Básicas Biomédicas \\ Facultad de Ciencias de la Salud \\ Universidad de Talca Av. Lircay s/n \\ Talca \\ CHILE
}

Email.iroa@utalca.cl

Recibido : 14-06-2016

Aceptado: 23-07-2016 